\title{
The structure of IL-11 Mutein suggests a surprising mechanism of inhibition
}

\author{
Riley D. Metcalfe ${ }^{1}$, Kaheina Aizel ${ }^{1}$, Paul M. Nguyen ${ }^{2,3}$, Courtney O. Zlatic ${ }^{1}$, Paul R. Gooley ${ }^{1}$, \\ Tracy L. Putoczki ${ }^{2,3}$, Michael D. W. Griffin ${ }^{1}$ \\ 1. Department of Biochemistry and Molecular Biology, Bio21 Molecular Science and \\ Biotechnology Institute, University of Melbourne, Parkville, Victoria 3010, Australia \\ 2. Inflammation Division, Walter and Eliza Hall Institute of Medical Research, Parkville, \\ Victoria 3052, Australia \\ 3. Department of Medical Biology, University of Melbourne, Parkville, Victoria 3010, Australia
}

Presenting author's email: rileydm@student.unimelb.edu.au

The multifunctional cytokine interleukin (IL) 11 has recently been shown to have roles in several diseases, including gastrointestinal cancer, where IL-11 signaling has been shown to be a therapeutic target. We have shown that IL-11 forms a hexameric signaling complex with its two receptors, the IL11 specific IL-11R $\alpha$, and the shared receptor gp 130. Preventing the assembly of the IL-11 signalling complex is an obvious strategy for IL-11 signalling inhibition.

We were interested in the structural mechanism of a very potent antagonistic mutant of IL-11, IL-11 Mutein [1]. IL-11 Mutein comprises a mutation in a gp130 binding site (IL-11 W147A $_{\mathrm{A}}$ ) which was proposed to prevent the assembly of the hexameric signalling complex, and a second set of mutations (IL-11 $1_{\text {PAIDY }}$, which were thought to increase affinity for IL-11R $\alpha$, enabling Mutein to outcompete IL11 for IL-11 R $\alpha$. We have shown that IL-11 and IL-11 Mutein have similar affinity for IL-11R $\alpha$, meaning that the suggested mechanism of action is not possible. We have further shown in vitro, that IL-11 Mutein abolishes IL-11 signalling, while IL-11 $1_{\mathrm{W} 168 \mathrm{~A}}$ permits limited IL-11 signalling, implying that the IL-11 $1_{\text {PAIDY }}$ mutations may further decrease affinity for gp130 over IL-11 W147A. To understand the structural basis of Mutein antagonism, we have solved the crystal structure of IL-11, IL-11 Mutein and IL-1 $1_{\mathrm{W} 147 \mathrm{~A}}$. These structures show that a loop in the vicinity of a gp130 binding site is displaced in IL11 Mutein compared to IL-11 $1_{\mathrm{W} 147 \mathrm{~A}}$, suggesting that alteration of loop dynamics form the structural basis for IL-11 Mutein antagonism. We are currently conducting molecular dynamics (MD) simulations of IL-11 and IL-11 mutants, to understand the nature of loop dynamics in both proteins.

\section{Reference:}

[1] Chun, G. L., Hartl, D., Matsuura, H., Dunlop, F. M., Scotney, P.D., Fabri, L.J ., Nash, A. D., Chen, N. Y., Tang, C. Y., Chen, Q., Homer, R. J., Baca, M. and Elias J. (2008) "Endogenous IL11 signaling is essential in Th2- and IL-13-induced inflammation and mucus production", Am. J. Respir. Cell Mol Biol. 39:739-746 